\title{
Separated spectra and operator inequalities
}

\author{
M. Sababheh*1 (D), H. R. Moradi ${ }^{2}$ \\ ${ }^{1}$ Department of Basic Sciences, Princess Sumaya University For Technology, Al Jubaiha, Amman 11941, \\ Jordan \\ ${ }^{2}$ Department of Mathematics, Payame Noor University, P.O. Box 19395-4697 Tehran, Iran
}

\begin{abstract}
The main goal of this article is to show that many inequalities that are not valid in operator theory become true if we add a separation condition on the spectra. The applications include showing how monotone functions behave like operator monotone functions and how the Choi-Davis inequality becomes valid for convex functions under this separation condition.
\end{abstract}

Mathematics Subject Classification (2020). 47A63, 26A51, 26D15, 26B25, 39B62

Keywords. operator order, separated spectra, convex functions, operator monotone functions

\section{Introduction}

Let $\mathcal{B}(\mathcal{H})$ denote the $C^{*}$-algebra of all bounded linear operators on a Hilbert space $\mathcal{H}$. When $\mathcal{H}$ is finite dimensional of dimension $n$, we identify $\mathcal{B}(\mathcal{H})$ with $\mathcal{M}_{n}$; the algebra of all complex $n \times n$ matrices. The identity operator in $\mathcal{B}(\mathcal{H})$ will be denoted by $\mathcal{J}_{\mathcal{H}}$. If $\mathcal{B}(\mathcal{H})=\mathcal{M}_{n}$, we use $\mathcal{J}_{n}$ for the identity operator. A linear mapping $\Phi: \mathcal{B}(\mathcal{H}) \rightarrow \mathcal{B}(\mathcal{K})$ is said to be positive if it preserves positive operators. That is, if $\Phi(A) \geq 0$ whenever $A \geq 0$. In this context, an operator $A \in \mathcal{B}(\mathcal{H})$ is said to be positive, and is denoted as $A \geq 0$, if $\langle A x, x\rangle \geq 0, \forall x \in \mathcal{H}$. If $A$ is positive and invertible, it is said to be positive definite and is denoted as $A>0$. If the positive linear mapping $\Phi: \mathcal{B}(\mathcal{H}) \rightarrow \mathcal{B}(\mathcal{K})$ satisfies the additional condition that $\Phi\left(\mathcal{J}_{\mathcal{H}}\right)=\mathcal{J}_{\mathcal{K}}$, then $\Phi$ is said to be unital (or normalized).

We shall use the notation $\operatorname{sp}(A)$ to denote the spectrum of the operator $A$, which is defined by

$$
\operatorname{sp}(A)=\left\{\lambda \in \mathbb{C}: A-\lambda \mathcal{J}_{\mathcal{H}} \text { is not invertible in } \mathcal{B}(\mathcal{H})\right\} .
$$

If $f: J \rightarrow \mathbb{R}$ is a given continuous function defined on the interval $J$, and if $A \in \mathcal{B}(\mathcal{H})$ is a self adjoint operator with spectrum contained in $J$, the Gelfand map $\varphi$ defines a $*-$ isometrically isomorphism between the set $C(\operatorname{sp}(A))$ of all continuous functions on $\operatorname{sp}(A)$ and the $C^{*}$-algebra $C^{*}(A)$ generated by $A$ together with $\mathcal{J}_{\mathcal{H}}$. Then $f(A)$ is defined by $f(A)=\varphi(f)$. This is usually referred to as functional calculus. When $A$ is compact self adjoint, so it has a spectral decomposition $A=\sum_{i=1}^{\infty} \lambda_{i} e_{i} \otimes e_{i}$, then $f(A)=\sum_{i=1}^{\infty} f\left(\lambda_{i}\right) e_{i} \otimes$ $e_{i}$. Recall that if $e \in \mathcal{H}$, then the tensor product $e \otimes e: \mathcal{H} \rightarrow \mathcal{H}$ is defined as $(e \otimes e)(x)=$

\footnotetext{
*Corresponding Author.

Email addresses: sababheh@yahoo.com (M. Sababheh), hrmoradi@mshdiau.ac.ir (H. Moradi)

Received: 04.05.2020; Accepted: 05.02.2021
} 
$\langle x, e\rangle$ e. In case $A \in \mathcal{M}_{n}$ is Hermitian, then $A=U \operatorname{diag}\left(\lambda_{i}\right) U^{*}$, for some unnitary matrix $U$ and a diagonal matrix $\operatorname{diag}\left(\lambda_{i}\right)$. In this case, $f(A)$ is simply $f(A)=U \operatorname{diag}\left(f\left(\lambda_{i}\right)\right) U^{*}$.

Having defined $f(A)$, for the self adjoint operator $A$, we cite the following lemma from [6]. This lemma helps to obtain operator inequalities from scalar ones.

Lemma 1.1. Let $A \in \mathcal{B}(\mathcal{H})$ be self adjoint and let $f$ and $g$ be continuous real valued functions such that $f(t) \geq g(t)$ for all $t \in \operatorname{sp}(A)$. Then $f(A) \geq g(A)$.

In this context, the order relation $A \leq B$ between the self adjoint operators $A, B$ means that $B-A \geq 0$.

Among the most important classes of functions related to operator inequalities are the operator monotone and the operator convex functions. A function $f: J \rightarrow \mathbb{R}$ is said to be operator monotone increasing (respectively decreasing) if $f(A) \leq f(B)$ (respectively $f(A) \geq f(B)$ ) whenever $A, B \in \mathcal{B}(\mathcal{H})$ are self adjoint operators with spectra in the interval $J$ and $A \leq B$. It must be noticed that if $f$ is an increasing function (in the scalar sense), it does not follow that $f$ is operator monotone increasing and the function $f(t)=t^{2}$ provides such an example, see [4, Example V.1.2., P. 113]. For brevity, an operator monotone increasing function is usually just called operator monotone.

On the other hand, a function $f: J \rightarrow \mathbb{R}$ will be called an operator convex (respectively operator concave) function if for all self adjoint operators $A, B \in \mathcal{B}(\mathcal{H})$, with spectra in $J$, one has

$$
f\left(\frac{A+B}{2}\right) \leq \frac{f(A)+f(B)}{2}
$$

(respectively $\left.f\left(\frac{A+B}{2}\right) \geq \frac{f(A)+f(B)}{2}\right)$. Again, a convex function (in the scalar sense) is not necessarily operator convex and the function $f(t)=t^{3}, t>0$ provides such an example [4, Example V.1.4., P. 114].

It should be noticed that convex (and operator convex) functions are always continuous on the interior of their domain.

Unlike scalar functions, operator monotony and operator convexity are strongly related as stated below. See [12, Theorem 2.4] and [2, Theorems 2.1, 3.1, 2.3, 3.7].

Proposition 1.2. Let $f:(0, \infty) \rightarrow[0, \infty)$ be continuous. Then

(1) $f$ is operator monotone decreasing if and only if $f$ is operator convex and $f(\infty)<\infty$.

(2) $f$ is operator monotone increasing if and only if $f$ is operator concave.

Finding conditions that make scalar increasing functions satisfy the operator monotony property or those properties that make scalar convex functions satisfy operator convexity have received a considerable attention in the literature as one can see in $[9,10]$.

Our main target in this work is to explore further those conditions. For this purpose, we begin with the following definition.

Definition 1.3. Let $A \in \mathcal{B}\left(\mathcal{H}_{1}\right)$ and $B \in \mathcal{B}\left(\mathcal{H}_{2}\right)$ be two self adjoint operators. We say that the spectra of $A$ and $B$ are separated if there exists a number $M$ such that

$$
\alpha \leq M \leq \beta, \forall \alpha \in \tau(A), \beta \in \tau(B) \quad \text { or } \quad \alpha \geq M \geq \beta, \forall \alpha \in \tau(A), \beta \in \tau(B),
$$

where the notation $\tau(X)$ is used to denote the smallest interval containing the spectrum of the self adjoint operator $X$.

Notice that the condition that the spectra are separated is more strict than the spectra being disjoint. Also, we observe that when $A$ and $B$ have separated spectra, then the intersection $\tau(A) \cap \tau(B)$ is either empty or a singleton.

Among the most interesting operator inequalities is the celebrated Choi-Davis inequality which states that

$$
f(\Phi(A)) \leq \Phi(f(A))
$$


for the self adjoint operator $A \in \mathcal{B}(\mathcal{H})$, the operator convex function $f: J \rightarrow \mathbb{R}$ and the unital positive linear mapping $\Phi$. Of course, $\operatorname{sp}(A) \subset J$. The inequality (1.1) is not true in general for a convex function $f$ that is not operator convex.

In [9, Theorem 1], an $n$-tuple version of (1.1) was given. For $n=1$, this result provides a convex version of (1.1), as follows.

Theorem 1.4 ([9, Theorem 1]). Let $A \in \mathcal{M}_{n_{1}}$ be Hermitian and let $\Phi: \mathcal{M}_{n_{1}} \rightarrow \mathcal{M}_{n_{2}}$ be a unital positive linear map such that $A$ and $\Phi(A)$ have separated spectra. If $f: J \rightarrow \mathbb{R}$ is a convex function on the interval $J$ and if $\tau(A), \tau(\Phi(A)) \subseteq J$, then

$$
f(\Phi(A)) \leq \Phi(f(A)) .
$$

We remark that in [9], the definition of separated spectra was not given, but the condition stated in that reference is equivalent to the spectra being separated.

Our discussion will show that the assumptions of Theorem 1.4 cannot hold except for trivial cases. So, this result becomes more valuable when $n>1$. See Proposition 3.3 and Remark 3.4 below.

Our main results include showing how separated spectra makes an increasing function behave like an operator monotone function and convex functions behave like operator convex functions.

In the sequel, $\lambda_{1}(X), \lambda_{2}(X), \cdots, \lambda_{n}(X)$, will denote the eigenvalues of the Hermitian matrix $X \in \mathcal{M}_{n}$ arranged so that $\lambda_{1}(X) \geq \lambda_{2}(X) \geq \cdots \geq \lambda_{n}(X)$.

\section{Separated spectra and monotone functions}

In this section we discuss separated spectra and their role in operator monotony. In the sequel, if $A \in \mathcal{B}(\mathcal{H})$ is self adjoint and if $M$ is a scalar, we understand the inequality $A \leq M$ as $A \leq M \mathcal{J}_{\mathcal{H}}$. Similar understanding applies for $m \leq A$, when $m$ is a scalar too.

Lemma 2.1. Let $f: J \rightarrow \mathbb{R}$ be an increasing continuous function on the interval $J$. If $A \in \mathcal{B}(\mathcal{H})$ is a self adjoint operator with spectrum in $J$, and if $A \leq M$ for some scalar $M \in J$, then $f(A) \leq f(M)$. On the other hand, if $m \leq A$ for some $m \in J$, then $f(m) \leq f(A)$.

Proof. It suffices to show the first inequality. So, assume that $A \leq M$ for some scalar $M \in J$. Since $f$ is increasing on $J$ and $M \in J$, it follows that

$$
f(t) \leq f(M) ; \forall t \in J, t \leq M .
$$

Now since $A \leq M$ and $\operatorname{sp}(A) \subset J$, it follows that $\operatorname{sp}(A) \subset(-\infty, M] \cap J:=J_{1}$; a sub-interval of $J$. From (2.1), we have $f(t) \leq f(M)$ for all $t \in J_{1}$. Finally, since $\operatorname{sp}(A) \subset J_{1}$, applying functional calculus (Lemma 1.1) for $t=A$ in the last inequality yields $f(A) \leq f(M)$; as desired.

Strongly related to our discussion, we present the following lemma, which presents a sufficient condition for the order $A \leq B$ or $B \leq A$.

Lemma 2.2. Let $A, B$ be two compact self adjoint operators in $\mathcal{B}(\mathcal{H})$ with separated spectra. Then either $A \leq B$ or $B \leq A$.

Proof. By the spectral decomposition, let $\left\{u_{k}\right\}$ and $\left\{v_{k}\right\}$ be two orthonormal bases of $\mathcal{H}$ such that

$$
A x=\sum_{k=1}^{\infty} \lambda_{k}\left\langle x, u_{k}\right\rangle u_{k} \text { and } B x=\sum_{k=1}^{\infty} \eta_{k}\left\langle x, v_{k}\right\rangle v_{k}
$$


and such that $A u_{k}=\lambda_{k} u_{k}$ and $B v_{k}=\eta_{k} v_{k}$. Noting that $\|x\|^{2}=\sum_{k=1}^{\infty}\left|\left\langle x, u_{k}\right\rangle\right|^{2}$, we have, for $x \neq 0$,

$$
\begin{aligned}
\frac{1}{\|x\|^{2}}\langle A x, x\rangle & =\frac{1}{\|x\|^{2}}\left\langle\sum_{k=1}^{\infty} \lambda_{k}\left\langle x, u_{k}\right\rangle u_{k}, \sum_{k=1}^{\infty}\left\langle x, u_{k}\right\rangle u_{k}\right\rangle \\
& =\sum_{k=1}^{\infty} \lambda_{k} \frac{\left|\left\langle x, u_{k}\right\rangle\right|^{2}}{\|x\|^{2}},
\end{aligned}
$$

which is a convex combination of the $\left\{\lambda_{k}\right\}$. Therefore,

$$
\frac{1}{\|x\|^{2}}\langle A x, x\rangle \in \tau(A) \text {. }
$$

Similarly, we conclude that

$$
\frac{1}{\|x\|^{2}}\langle B x, x\rangle \in \tau(B) \text {. }
$$

Now since $\tau(A)$ and $\tau(B)$ are separated, we infer that

$$
\alpha \leq \beta \forall \alpha \in \tau(A), \beta \in \tau(B) \text { or } \alpha \geq \beta \forall \alpha \in \tau(A), \beta \in \tau(B) .
$$

Consequently,

$$
\text { either } \frac{1}{\|x\|^{2}}\langle A x, x\rangle \leq \frac{1}{\|x\|^{2}}\langle B x, x\rangle \forall x \in \mathcal{H} \text { or } \frac{1}{\|x\|^{2}}\langle A x, x\rangle \geq \frac{1}{\|x\|^{2}}\langle B x, x\rangle \forall x \in \mathcal{H} \text {. }
$$

But this latter conclusion leads to the fact that either $\langle A x, x\rangle \leq\langle B x, x\rangle$ for all $x \in \mathcal{H}$ or $\langle A x, x\rangle \geq\langle B x, x\rangle$ for all $x \in \mathcal{H}$, which yields $A \leq B$ or $B \leq A$, as required.

So, in some cases, one can use Lemma 2.2 to determine if $A \leq B$ (of course, or $B \leq A$ ). Notice that determining whether $A \leq B$ or not needs to determine positivity of $B-A$. However, Lemma 2.2 presents a very special case, where one can determine this order by finding the spectra of $A$ and $B$.

After finding the spectra of both $A$ and $B$, the intervals $\tau(A)$ and $\tau(B)$ become clear. If the two intervals are separated, then Lemma 2.2 says that either $A \leq B$ or $B \leq A$. To decide, we just compare $\lambda_{1}(A)$ and $\lambda_{1}(B)$. If, for example, $\lambda_{1}(A) \leq \lambda_{1}(B)$, then we have the order $A \leq B$.

The next theorem shows how separated spectra affect operator monotony.

Theorem 2.3. Let $f: J \rightarrow \mathbb{R}$ be a continuous increasing function and let $A, B \in \mathcal{B}(\mathcal{H})$ be two self adjoint operators with spectra in $J$. If $A$ and $B$ have separated spectra and if $A \leq B$, then $f(A) \leq f(B)$.

Proof. Since $\tau(A)$ and $\tau(B)$ are separated and since $A \leq B$, it follows that there is a scalar $s$ such that

$$
t_{1} \leq s \leq t_{2}
$$

for all $t_{1} \in \tau(A), t_{2} \in \tau(B)$. Clearly $s \in J$. Now applying Lemma 2.1, we obtain $f(A) \leq$ $f(s) \leq f(B)$, which completes the proof.

Corollary 2.4. Let $A, B$ be two self adjoint operators in $\mathcal{B}(\mathcal{H})$ and let $M$ be a scalar such that $A \leq M \leq B$. If $f: J \rightarrow \mathbb{R}$ is a continuous increasing function on the interval $J$ that contains the spectra of $A, B$. Then $f(A) \leq f(B)$.

Proof. Since $A \leq M \leq B$, we infer that $\tau(A) \subset(-\infty, M]$ and $\tau(B) \subset[M, \infty)$. This means that $A$ and $B$ have separated spectra. From Theorem 2.3 we get the desired inequality.

In the next result, we show a monotone result without having separated spectra. We emphasize that this result was shown in [11] using a completely different argument and approach. Our proof depends on separating the spectra. 
Corollary 2.5. Let $f:(0, \infty) \rightarrow \mathbb{R}$ be a continuous increasing function and let $A, B$ be positive definite matrices in $\mathcal{M}_{n}$ such that $A \leq B$. Then

$$
f\left(\frac{\lambda_{n}(A)}{\lambda_{1}(A)} A\right) \leq f(B) .
$$

Proof. Notice that since $A, B>0$ and $A \leq B$, then

$$
\lambda_{j}(A) \leq \lambda_{j}(B), \forall j=1, \cdots, n .
$$

From this, it follows that

$$
\lambda_{1}\left(\frac{\lambda_{n}(A)}{\lambda_{1}(A)} A\right)=\frac{\lambda_{n}(A)}{\lambda_{1}(A)} \lambda_{1}(A)=\lambda_{n}(A) \leq \lambda_{n}(B) .
$$

But since $\lambda_{n}(B) \leq \lambda_{j}(B)$ for $1 \leq j \leq n$, the latter inequality gives

$$
\begin{aligned}
\lambda_{j}\left(\frac{\lambda_{n}(A)}{\lambda_{1}(A)} A\right) & \leq \lambda_{1}\left(\frac{\lambda_{n}(A)}{\lambda_{1}(A)} A\right) \\
& =\lambda_{n}(A) \leq \lambda_{n}(B) \leq \lambda_{j}(B) ; 1 \leq j \leq n .
\end{aligned}
$$

From this, we infer

$$
\tau\left(\frac{\lambda_{n}(A)}{\lambda_{1}(A)} A\right)=\left[\frac{\lambda_{n}^{2}(A)}{\lambda_{1}(A)}, \lambda_{n}(A)\right] .
$$

But also we have $\tau(B)=\left[\lambda_{n}(B), \lambda_{1}(B)\right]$, and this gives

$$
\tau\left(\frac{\lambda_{n}(A)}{\lambda_{1}(A)} A\right) \cap \tau(B)=\emptyset
$$

except for the case when $\lambda_{n}(A)=\lambda_{n}(B)$, which then assures that $\tau\left(\frac{\lambda_{n}(A)}{\lambda_{1}(A)} A\right) \cap \tau(B)$ is a singleton. By Lemma 2.2 we have

$$
\text { either } \frac{\lambda_{n}(A)}{\lambda_{1}(A)} A \leq B \text { or } B \leq \frac{\lambda_{n}(A)}{\lambda_{1}(A)} A \text {. }
$$

However, since $\lambda_{j}\left(\frac{\lambda_{n}(A)}{\lambda_{1}(A)} A\right) \leq \lambda_{j}(B)$, it follows that $\frac{\lambda_{n}(A)}{\lambda_{1}(A)} A \leq B$. By Theorem 2.3, the desired inequality follows.

Similarly one can show the following better estimate than the one in Corollary 2.5.

Corollary 2.6. Let $f:(0, \infty) \rightarrow \mathbb{R}$ be a continuous increasing function and let $A, B>0$ be such that $A \leq B$. Then

$$
f\left(\frac{\lambda_{n}(B)}{\lambda_{1}(A)} A\right) \leq f(B) .
$$

In the following result, we present a different, but a related condition that guarantees operator monotony. Then we present some applications.

Proposition 2.7. Let $A, B \in \mathcal{M}_{n}$ be two commuting Hermitian matrices such that $A \leq B$. If $f: J \rightarrow \mathbb{R}$ is a continuous increasing function on the interval $J$ that contains the spectra of both $A, B$, then $f(A) \leq f(B)$.

Proof. This follows from the fact that two commuting Hermitian matrices are simultaneously diagonalizable.

Now we present an interesting application of Proposition 2.7. We know that (see [11]) if $\Phi$ is a unital positive linear map and $m \leq A \leq M$, then

$$
\Phi(A) \sharp \Phi\left(A^{-1}\right) \leq \frac{M+m}{2 \sqrt{M m}},
$$


where $A \sharp B=A^{\frac{1}{2}}\left(A^{-\frac{1}{2}} B A^{-\frac{1}{2}}\right)^{\frac{1}{2}} A^{\frac{1}{2}}$. In [8], it is shown that

$$
\Phi\left(A^{-1}\right)^{2} \leq\left(\frac{(m+M)^{2}}{4 m M}\right)^{2} \Phi(A)^{-2},
$$

as a squared version of (2.3). In fact, from (2.4) we infer

$$
\Phi(A)^{2} \sharp \Phi\left(A^{-1}\right)^{2} \leq \frac{(m+M)^{2}}{4 m M} .
$$

In the next result, we use Proposition 2.7 to obtain a refinement of (2.5). In the sequel, $\|\cdot\|$ denotes the usual operator norm.

Corollary 2.8. Let $A \in \mathcal{M}_{n}$ be a positive definite matrix and let $\Phi$ be a positive unital linear map on $\mathcal{M}_{n}$. Then

$$
\begin{aligned}
\Phi(A)^{2} \sharp \Phi\left(A^{-1}\right)^{2} & \leq\left\|\Phi(A)^{2} \sharp \Phi\left(A^{-1}\right)^{2}\right\| \\
& \leq\left\|\left(\Phi(A) \sharp \Phi\left(A^{-1}\right)\right)^{2}\right\| \\
& \leq \frac{(M+m)^{2}}{4 M m} .
\end{aligned}
$$

Proof. Notice that the two matrices

$$
\Phi(A) \sharp \Phi\left(A^{-1}\right) \quad \text { and } \quad \frac{M+m}{2 \sqrt{M m}} \mathcal{J}_{n}
$$

commute. Therefore, with $f(t)=t^{2}$, Proposition 2.7 can be applied to obtain

$$
\left(\Phi(A) \sharp \Phi\left(A^{-1}\right)\right)^{2} \leq \frac{(M+m)^{2}}{4 M m} \mathcal{J}_{n}
$$

from (2.3). Now, from Ando-Hiai inequality [1] it follows that

$$
\left\|\Phi(A)^{2} \sharp \Phi\left(A^{-1}\right)^{2}\right\| \leq\left\|\left(\Phi(A) \sharp \Phi\left(A^{-1}\right)\right)^{2}\right\| \leq \frac{(M+m)^{2}}{4 M m} .
$$

On the other hand, since $\Phi(A)^{2} \sharp \Phi\left(A^{-1}\right)^{2}$ is a positive operator, we have

$$
\Phi(A)^{2} \sharp \Phi\left(A^{-1}\right)^{2} \leq\left\|\Phi(A)^{2} \sharp \Phi\left(A^{-1}\right)^{2}\right\| .
$$

Combining (2.6) and (2.7), we get the desired inequalities.

To present the next application of Proposition 2.7, we recall that for the positive definite matrices $A, B$ and the operator monotone function $f:(0, \infty) \rightarrow(0, \infty)$, the binary operation $\sigma_{f}$ defined by

$$
A \sigma_{f} B=A^{\frac{1}{2}} f\left(A^{-\frac{1}{2}} B A^{-\frac{1}{2}}\right) A^{\frac{1}{2}}
$$

is an operator mean, provided that $f(1)=1$. In this context, we remind the reader that a binary operation $\sigma$ on the cone of positive definite matrices is said to be an operator mean if it satisfies the following conditions [7] for the positive definite matrices $A, B, C, D$ :

- $A \leq C$ and $B \leq D$ imply $A \sigma B \leq C \sigma D$.

- $C(A \sigma B) C=(C A C) \sigma(C B C)$.

- $A_{n} \downarrow_{n} A$ and $B_{n} \downarrow_{n} B$ imply $\left(A_{n} \sigma B_{n}\right) \downarrow_{n}(A \sigma B)$.

- $\mathcal{J}_{n} \sigma \mathcal{J}_{n}=\mathcal{J}_{n}$. 
When $f$ is not operator monotone, $\sigma_{f}$ does not satisfy some of the desired properties for an operator mean. It is well known that when $f$ is operator monotone such that $f(1)=1$, the condition $B \leq A$ implies $A \sigma_{f} B \leq A$. Also, it is well known that when $\sigma_{f}$ is symmetric in the sense that $A \sigma_{f} B=B \sigma_{f} A$, then $A \sigma_{f} B \geq B$. We refer the reader to [3]. In the next result, we show these properties for a monotone function $f$ that is not necessarily operator monotone.

Corollary 2.9. Let $A, B \in \mathcal{M}_{n}$ be positive definite. If $f:(0, \infty) \rightarrow(0, \infty)$ is a continuous increasing function such that $f(1)=1$ and $B \leq A$, then $A \sigma_{f} B \leq A$. Further, if $A \sigma_{f} B=$ $B \sigma_{f} A$, then $B \leq A \sigma_{f} B$.

Proof. Since $B \leq A$, we have $A^{-\frac{1}{2}} B A^{-\frac{1}{2}} \leq \mathcal{J}_{n}$. Proposition 2.7 gives $f\left(A^{-\frac{1}{2}} B A^{-\frac{1}{2}}\right) \leq$ $f\left(\mathcal{J}_{n}\right)=\mathcal{J}_{n}$. Now conjugating with $A^{\frac{1}{2}}$ yields the first desired inequality. For the second inequality, we observe that the condition $B \leq A$ implies $\mathcal{J}_{n} \leq B^{-\frac{1}{2}} A B^{-\frac{1}{2}}$, which means $\mathcal{J}_{n}=f\left(\mathcal{J}_{n}\right) \leq f\left(B^{-\frac{1}{2}} A B^{-\frac{1}{2}}\right)$. Conjugating this inequality with $B^{\frac{1}{2}}$ gives $B \leq B \sigma_{f} A$, meaning that $B \leq A \sigma_{f} B$ since $\sigma_{f}$ is symmetric.

\section{Separated spectra and convex functions}

Let $f: J \rightarrow \mathbb{R}$ be a function defined on the interval $J$ and let $A$ be a self adjoint operator in $\mathcal{B}(\mathcal{H})$. If $\tau(A)=[m, M]$, we use the notations

$$
a_{f, A}=\frac{f(M)-f(m)}{M-m}, b_{f, A}=\frac{M f(m)-m f(M)}{M-m} .
$$

Theorem 3.1. Let $A \in \mathcal{M}_{n_{1}}$ be Hermitian and let $\Phi: \mathcal{M}_{n_{1}} \rightarrow \mathcal{M}_{n_{2}}$ be a positive linear map such that $A$ and $\Phi(A)$ have separated spectra. If $f: J \rightarrow \mathbb{R}$ is a convex (continuous) function on the interval $J$ containing $\tau(A)$ and $\tau(\Phi(A))$, then

$$
f(\Phi(A)) \leq \Phi(f(A))+b_{f, \Phi(A)}\left(\mathcal{J}_{n_{2}}-\Phi\left(\mathcal{J}_{n_{1}}\right)\right) .
$$

Proof. Assume that $\tau(\Phi(A))=[m, M]$ and let $g(t)=a_{f, \Phi(A)} t+b_{f, \Phi(A)}$ be the line equation through $(m, f(m))$ and $(M, f(M))$. Then

$$
f(t) \leq a_{f, \Phi(A)} t+b_{f, \Phi(A)} ; \forall t \in[m, M] .
$$

Applying functional calculus argument to the above inequality with $t=\Phi(A)$, we get

$$
f(\Phi(A)) \leq a_{f, \Phi(A)} \Phi(A)+b_{f, \Phi(A)} \mathcal{J}_{n_{2}} .
$$

On the other hand, since $\tau(A)$ and $\tau(\Phi(A))$ are separated, we may apply functional calculus argument to the inequality

$$
f(t) \geq a_{f, \Phi(A)} t+b_{f, \Phi(A)} ; \forall t \in J \backslash[m, M],
$$

using $t=A$ to obtain

$$
f(A) \geq a_{f, \Phi(A)} A+b_{f, \Phi(A)} \mathcal{J}_{n_{1}},
$$

which gives, upon applying $\Phi$,

$$
\Phi(f(A)) \geq a_{f, \Phi(A)} \Phi(A)+b_{f, \Phi(A)} \Phi\left(\mathcal{J}_{n_{1}}\right) .
$$

Then the desired inequality follows by combiing (3.1) and (3.2).

The following converse of Theorem 3.1 can be obtained by applying the same ideas as in Theorem 3.1, but taking $g$ as the secant of $f$ over the interval $\tau(A)$ instead of $\tau(\Phi(A))$. That is, if $\tau(A)=[m, M]$, then $g(t)=a_{f, A} t+b_{f, A}$; the secant through the points $(m, f(m))$ and $(M, f(M))$. 
Theorem 3.2. Let $A \in \mathcal{M}_{n_{1}}$ be Hermitian and let $\Phi: \mathcal{M}_{n_{1}} \rightarrow \mathcal{M}_{n_{2}}$ be a positive linear map such that $A$ and $\Phi(A)$ have separated spectra. If $f: J \rightarrow \mathbb{R}$ is a convex (continuous) function on the interval $J$ and if $\tau(A), \tau(\Phi(A)) \subseteq J$, then

$$
\Phi(f(A)) \leq f(\Phi(A))+b_{f, A}\left(\Phi\left(\mathcal{J}_{n_{1}}\right)-\mathcal{J}_{n_{2}}\right) .
$$

Notice that if $\Phi$ is unital in Theorems 3.1 and 3.2, we deduce that

$$
f(\Phi(A))=\Phi(f(A)) .
$$

At first glance, this conclusion seems to be a contradiction to the case $n=1[9$, Theorem 1] stated in Theorem 1.4 above. In the next proposition, we clarify this, where we show that when $\Phi$ is unital, $A$ and $\Phi(A)$ cannot have separated spectra except for trivial cases that allow the identity $f(\Phi(A))=\Phi(f(A))$ to hold.

Proposition 3.3. Let $A \in \mathcal{M}_{n}$ be Hermitian and let $\Phi: \mathcal{M}_{n} \rightarrow \mathcal{M}_{m}$ be a unital positive linear mapping. Then $A$ and $\Phi(A)$ have separated spectra if and only if either $\Phi(A)=$ $\lambda_{1}(A) \mathcal{J}_{n}$ or $\Phi(A)=\lambda_{n}(A) \mathcal{J}_{n}$.

Proof. Assume first that $\Phi(A)=\lambda_{1}(A) \mathcal{J}_{n}$ or $\Phi(A)=\lambda_{n}(A) \mathcal{J}_{n}$. Then $\tau(\Phi(A))=\left\{\lambda_{1}(A)\right\}$ or $\tau(\Phi(A))=\left\{\lambda_{n}(A)\right\}$, and in both cases $A$ and $\Phi(A)$ have separated spectra.

On the other hand, assume that $A$ and $\Phi(A)$ have separated spectra. We know that, since $\Phi$ is unital,

$$
\lambda_{n}(A) \mathcal{J}_{n} \leq A \leq \lambda_{1}(A) \mathcal{J}_{n} \Rightarrow \lambda_{n}(A) \mathcal{J}_{n} \leq \Phi(A) \leq \lambda_{1}(A) \mathcal{J}_{n} .
$$

But the latter inequality assures that $\tau(\Phi(A)) \subset\left[\lambda_{n}(A), \lambda_{1}(A)\right]$. Now since, by assumption, $\tau(A)$ and $\tau(\Phi(A))$ have separated spectra and since $\tau(\Phi(A)) \subset\left[\lambda_{n}(A), \lambda_{1}(A)\right]$, we must have $\tau(\Phi(A))=\left\{\lambda_{n}(A)\right\}$ or $\tau(\Phi(A))=\left\{\lambda_{1}(A)\right\}$, upon which we infer that $\sigma(\Phi(A))=$ $\left\{\lambda_{n}(A)\right\}$ or $\sigma(\Phi(A))=\left\{\lambda_{n}(A)\right\}$. Since $\Phi(A)$ is Hermitian, it follows that $\Phi(A)=\lambda_{1}(A) \mathcal{J}_{n}$ or $\Phi(A)=\lambda_{n}(A) \mathcal{J}_{n}$, completing the proof.

In the following remark, we show the same conclusion as Proposition 3.3 but for the special case when $\Phi$ is a state.

Remark 3.4. Notice that if $\Phi: \mathcal{M}_{n} \rightarrow \mathbb{C}$ is a state; that is $\Phi(A)=\langle A x, x\rangle$ for a specific unit vector $x$, then using the spectral decomposition $A x=\sum_{k=1}^{n} \lambda_{k}\left\langle x, u_{k}\right\rangle u_{k}$, we have

$$
\begin{aligned}
\Phi(A) & =\langle A x, x\rangle \\
& =\sum_{k=1}^{n} \lambda_{k}\left|\left\langle x, u_{k}\right\rangle\right|^{2} \in \tau(A),
\end{aligned}
$$

where the last inclusion follows from the fact that $x$ is a unit vector, which in turns assures that the above sum is a convex combination of the $\left\{\lambda_{k}\right\}$. This shows that $\Phi(A) \in \tau(A)$ and hence, when $\Phi$ is a state, $A$ and $\Phi(A)$ do not have separated spectra unless $\Phi(A)=\lambda_{n}(A) \mathcal{J}_{n}$ or $\Phi(A)=\lambda_{1}(A) \mathcal{J}_{n}$.

We conclude this article with the following subadditive inequality for convex functions. Recall that a concave function $f:(0, \infty) \rightarrow(0, \infty)$ is subadditive in the sense that

$$
f(a+b) \leq f(a)+f(b) \text {. }
$$

This inequality does not hold for operator concave functions. That is, if $f:(0, \infty) \rightarrow$ $(0, \infty)$ is an operator concave function and if $A$ and $B$ are two positive definite operators in $\mathcal{B}(\mathcal{H})$, then it does not follow that

$$
f(A+B) \leq f(A)+f(B) .
$$

In [3], it is shown that

$$
\|f(A+B)\|_{u} \leq\|f(A)+f(B)\|_{u},
$$


for the operator concave function $f:(0, \infty) \rightarrow(0, \infty)$ and the positive definite matrices $A, B \in \mathcal{M}_{n}$, where $\|\cdot\|_{u}$ is any unitarily invariant norm. Later, Bourin and Uchiyama [5] proved the validity of (3.5) for the concave $f$ (not necessarily operator concave). We also refer the reader to [10] for further discussion in this direction. In the next result, we prove a subadditive inequality for convex functions, under the separated spectra condition.

Theorem 3.5. Let $A, B \in \mathcal{B}(\mathcal{H})$ be such that $A$ and $A+B$ as well as $B$ and $A+B$ have separated spectra. If $f: J \rightarrow \mathbb{R}$ is a convex (continuous) function on the interval $J$ containing the spectra of $A, B, A+B$, then

$$
f(A+B) \leq f(A)+f(B)-b_{f, A+B} \mathcal{J}_{\mathcal{H}} .
$$

Proof. Assume that $\tau(A+B)=[m, M]$ and consider the line $g(t)=a_{f, A+B} t+b_{f, A+B}$. Since, on $[m, M], f(t) \leq g(t)$, applying functional calculus argument to $A+B$ gives

$$
f(A+B) \leq a_{f, A+B}(A+B)+b_{f, A+B} \mathcal{J}_{\mathcal{H}} .
$$

On the other hand, since $A$ and $B$ both have separated spectra with $A+B$, it follows that

$$
f(A) \geq a_{f, A+B} A+b_{f, A+B} \mathcal{J}_{\mathcal{H}} \quad \text { and } \quad f(B) \geq a_{f, A+B} B+b_{f, A+B} \mathcal{J}_{\mathcal{H}},
$$

which imply

$$
f(A)+f(B) \geq a_{f, A+B}(A+B)+2 b_{f, A+B} \mathcal{J}_{\mathcal{H}} .
$$

Then the desired inequality follows by combining (3.6) and (3.7).

\section{References}

[1] T. Ando and F. Hiai, Log-majorization and complementary Golden-Thompson type inequalities, Linear Alg. Appl. 197/198, 113-131, 1994.

[2] T. Ando and F. Hiai, Operator log-convex functions and operator means, Math. Ann. 350 (3), 611-630, 2011.

[3] T. Ando and X. Zhan, Norm inequalities related to operator monotone functions, Math. Ann. 315, 771-780, 1999.

[4] R. Bhatia, Matrix analysis, Springer-Verlag, New York, 1997.

[5] J.C. Bourin and M. Uchiyama, A matrix subadditivity inequality for $f(A+B)$ and $f(A)+f(B)$, Linear Algebra Appl. 423, 512-518, 2007.

[6] T. Furuta, J. Micic Hot, J. Pecaric and Y. Seo, Mond-Pecaric Method in Operator Inequalities, Monographs in Inequalities 1, Element, Zagreb, 2005.

[7] F. Kubo and T. Ando, Means of positive linear operators, Math. Ann. 246, 205-224, 1980.

[8] M. Lin, On an operator Kantorovich inequality for positive linear maps, J. Math. Anal. Appl. 402, 127-132, 2013.

[9] J. Mićić, Z. Pavić and J. Pečarić, Jensen's inequality for operators without operator convexity, Linear Algebra Appl. 434, 1228-1237, 2011.

[10] H.R. Moradi, Z. Heydarbeygi and M. Sababheh, Subadditive inequalities for operators, Math. Ineq. Appl. 23 (1), 317-327, 2020.

[11] R. Nakamoto and M. Nakamura, Operator mean and Kantorovich inequality, Math. Japon. 44 (3), 495-498, 1996.

[12] M. Uchiyama, Operator monotone functions, positive definite kernels and majorization, Proc. Amer. Math. Soc. 138 (11), 3985-3996, 2010. 